\title{
Molecular survey of Enterocytozoon bieneusi in sheep and goats in China
}

Ke Shi, Mengjie Li, Xiaoxing Wang, Junqiang Li, Md Robiul Karim, Rongjun Wang, Longxian Zhang,

Fuchun Jian and Changshen Ning*

\begin{abstract}
Background: Over recent years, several studies have conducted genotyping of Enterocytozoon bieneusi in various hosts worldwide using sequence analysis of the ribosomal internal transcribed spacer (ITS), however, relatively little is known about E. bieneusi in sheep and goats in China. Therefore this research was conducted to understand the prevalence and genotype distribution of E. bieneusi in farmed sheep and goats in China.

Results: A total of 1025 fecal specimens from farmed animals in various geographic areas were collected. Overall, PCR and sequence analysis of the ITS detected E. bieneusi in $34.4 \%$ (353/1025) of isolates; of which the prevalence in goats was $28.8 \%$ (176/611) and in sheep was $42.8 \%$ (177/414). Phylogenetic analysis of ITS sequences identified 42 genotypes (nine known and 33 novel ones). These consisted of four known genotypes ( $D, K I N-1$, EbpC, and F) and 10 novel genotypes (CHG6, CHG7, CHG9, CHG19, CHG23, CHG25, CHS5 and CHS10-CHS12) which all belonged to the so-called zoonotic group 1. A further four known genotypes (CD6, COS-I, BEB6, and J) and 22 novel genotypes (CHG1-CHG3, CHG5, CHG8, CHG10-CHG14, CHG16-CHG18, CHG20, CHG22, CHG24, CHS3, CHS4 and CHS6-CHS9) formed a clade within the group 2. One novel genotype (CHG21) was clustered in the group 9 with the genotype CM4.
\end{abstract}

Conclusions: E. bieneusi is highly prevalent, widely distributed, and genetically diverse in Chinese farmed goats and sheep. Some of the genotypes identified are potentially zoonotic.

Keywords: Enterocytozoon bieneusi, Goats, Sheep, ITS gene

\section{Background}

Microsporidia are a diverse group of obligate intracellular pathogens related to fungi [1]. To date, approximately 1300 species belonging to 160 microsporidian genera are known to infect invertebrate and vertebrate hosts, including humans $[2,3]$. Among the 14 microsporidian species infectious to humans, Enterocytozoon bieneusi is regarded as the most commonly identified microsporidian species in humans $[4,5]$. Thus far, in addition to humans, $E$. bieneusi has been detected in domestic animals such as cattle, sheep, goats, rabbits, pigs, dogs, cats, and horses [6-14], and in wild animals such as raccoons, muskrats, beavers, foxes, otters, nonhuman primates (NHPs), llamas, guinea pigs, deer and snakes [15-21] and birds [22].

\footnotetext{
* Correspondence: nnl1986@163.com

College of Animal Science and Veterinary Medicine, Henan Agricultural University, Zhengzhou 450002, China
}

E. bieneusi spores are morphologically indistinguishable and in vitro cultivation methods for this pathogen have not been developed. Therefore, molecular methods are required to identify genotypes within the species that infect humans, animals or both [3]. Until now, the internal transcribed spacer (ITS) of the rRNA gene has been used widely for species identification and genotyping of E. bieneusi isolates [11, 23, 24] and more than 200 E. bieneusi ITS genotypes have been identified [3-12]. Previous studies have reported that some genotypes are host-specific, whereas others are zoonotic because of their capability to infect both humans and animals [15, 16, 25-28]. Currently, over 30 countries have been surveyed to gather data on the molecular epidemiology of E. bieneusi in humans and other animals [29]. In China, this pathogen has been detected in HIV-positive and -negative patients, children, non-human primates (NHPs), dogs, cats, pigs, cows, sheep, goats, deer and snakes $[8,9,11,12,20,21,30-37]$. Moreover, this parasite has been identified in wastewater in 
several Chinese cities [38]. However, few studies have been conducted on E. bieneusi infections in sheep and goats in China [37]. In the present study, a large number of farms were surveyed to gain a better understanding of the prevalence and genotype distribution of $E$. bieneusi in sheep and goats in various parts of China.

\section{Methods}

\section{Ethics statement}

This research was conducted according to the Chinese Laboratory Animal Administration Act (1988) after review and approval of its protocol by the Research Ethics Committee of Henan Agricultural University. Appropriate permission was obtained from the farm owners before collection of fecal specimens from their sheep and goats.

\section{Fecal specimen collection}

A total of 1025 fresh fecal samples were collected from sheep and goats in Henan, Heilongjiang, Liaoning, Yunnan, Anhui, and Shaanxi Provinces and Chongqing city between March 2011 and October 2013 (Table 1 and Additional file
1). Specimens were collected either directly from the rectum or were collected in sterile plastic containers during defecation (without the fecal sample having contact with the ground). One fecal specimen per sheep or goat was collected in a container marked with the breed, gender, age, and feeding habits of the animal. No obvious clinical symptoms were observed during specimen collection, which were then transported in an icebox to the laboratory for examination.

\section{Specimen preparation}

The fecal specimen from each container was transferred to a sterile beaker immediately on arrival at the laboratory, soaked for thirty minutes at room temperature in $30 \mathrm{ml}$ of tap water, and then stirred with a clean stick to ensure thorough mixing. Each mixture was then put through a 7.62-cm-diameter sieve (pore size $45 \mu \mathrm{m}$ ) and concentrated by centrifugation at $3500 \mathrm{r} \cdot \mathrm{min}^{-1}$ for 5 min. The concentrated fecal material was stored in $2.5 \%$ potassium dichromate solution at $4{ }^{\circ} \mathrm{C}$ prior to DNA extraction.

Table 1 Prevalence and genotype distribution of E. bieneusi isolates from goat and sheep farms from different Provinces

\begin{tabular}{|c|c|c|c|c|c|}
\hline Species & Geographic source & No. of farms & No. of specimens & No. (\%) of positive specimens & ITS genotype(s) $\left(n^{\mathrm{a}}\right)$ \\
\hline \multirow[t]{6}{*}{ Goats } & Henan Province & 10 & 343 & $113(32.9)$ & $\begin{array}{l}\mathrm{BEB6}(22), \mathrm{E}(3), \mathrm{F}(2), \mathrm{KIN}-1(2), \mathrm{D}(2), \mathrm{J}(1), \mathrm{COS}-\mathrm{I}(1), \\
\mathrm{CD6}(4), \mathrm{CHG} 1(13), \mathrm{CHG} 2(6), \mathrm{CHG} 3(10), \mathrm{CHG} 5(3), \\
\mathrm{CHG6}(1), \mathrm{CHG} 7(1), \mathrm{CHG} 8(1), \mathrm{CHG} 9(1), \mathrm{CHG} 10(1), \\
\mathrm{CHG} 11(1), \mathrm{CHG} 13(1), \mathrm{CHG} 18(1), \mathrm{CHG} 20(1), \mathrm{CHG} 21 \\
\text { (1), CHG22(1), CHG23(1) ), CHG25(1); }\end{array}$ \\
\hline & Yunnan Province & 4 & 134 & $30(22.4)$ & 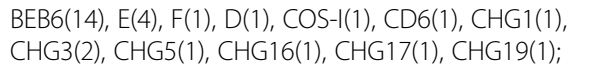 \\
\hline & Anhui Province & 1 & 80 & $6(7.5)$ & BEB6(1), CHG5(1), CHG3(1); \\
\hline & Chongqing city & 1 & 8 & $5(62.5)$ & CHG1(2), CHG3(1), CD6(1), CHG12(1); \\
\hline & Shaanxi Province & 1 & 46 & $22(47.8)$ & $\begin{array}{l}\mathrm{BEB6}(4), \mathrm{E}(1), \mathrm{F}(1), \mathrm{CHG} 1(3), \mathrm{CHG} 3(3), \mathrm{CD} 6(3), \\
\mathrm{CHG} \text { (2), CHG14(1), CHG16(1), CHG24(1); }\end{array}$ \\
\hline & Total & 17 & 611 & $176(28.8)$ & $\begin{array}{l}\text { BEB6(41), D(3), E(8), F(4), KIN-1(2), J(1), CHG1(19), } \\
\text { CHG2(6), CHG3(17), CD6(9), CHG5(7), CHG6(1), } \\
\text { CHG7(1), CHG8(1), CHG9(1), CHG10(1), CHG11(1), } \\
\text { CHG12(1), CHG13(1), CHG14(1), COS-I(2), CHG16(2), } \\
\text { CHG17(1), CHG18(1), CHG19(1), CHG20(1), CHG21(1), } \\
\text { CHG22(1), CHG23(1), CHG24(1), CHG25(1); }\end{array}$ \\
\hline \multirow[t]{4}{*}{ Sheep } & Henan Province & 4 & 310 & $161(51.9)$ & $\begin{array}{l}\mathrm{BEB} 6(53), \mathrm{COS}-1(12), \mathrm{CM} 4(1), \mathrm{CHG} 3(5), \mathrm{CHS3}(2), \\
\mathrm{CHS4}(1), \mathrm{CHS5}(1), \mathrm{CHS6}(1), \mathrm{CHS10}(1), \mathrm{CHS12}(1) ;\end{array}$ \\
\hline & Liaoning Province & 1 & 64 & $6(9.4)$ & $\mathrm{BEB6}(3)$ \\
\hline & $\begin{array}{l}\text { Heilongjiang } \\
\text { Province }\end{array}$ & 1 & 40 & $10(25.0)$ & $\begin{array}{l}\text { BEB6(4), COS-I(2), CHS7(1), CHS8(1), CHS9(1), } \\
\text { CHS11(1); }\end{array}$ \\
\hline & Total & 6 & 414 & $177(42.8)$ & $\begin{array}{l}\text { BEB6(60), COS-I(14), CHG3(5), CM4(1), CHS3(2), } \\
\text { CHS4(1), CHS5(1), CHS6(1), CHS7(1), CHS8(1), } \\
\text { CHS9(1)CHS10(1), CHS11(1), CHS12(1); }\end{array}$ \\
\hline \multicolumn{2}{|c|}{ All the Total } & 23 & 1025 & $353(34.4)$ & $\begin{array}{l}\text { F(4), KIN-1(2), J(1), CHG1(19), CHG2(6), CHG3(22), } \\
\text { CD6(9), CHG5(7), CHG6(1), CHG7(1), CHG8(1), } \\
\text { CHG9(1)CHG10(1), CHG11(1), CHG12(1), CHG13(1), } \\
\text { CHG14(1), COS-I(16), CHG16(2), CHG17(1),CHG18(1), } \\
\text { CHG19(1), CHG20(1), CHG21(1), CHG22(1), CHG23(1), } \\
\text { CHG24(1), CHG25(1), CM4(1), CHS3(2), CHS4(1), } \\
\text { CHS5(1), CHS6(1), CHS7(1), CHS8(1), CHS9(1), } \\
\text { CHS10(1), CHS11(1), CHS12(1), BEB6(101), D(3), E(8) }\end{array}$ \\
\hline
\end{tabular}




\section{DNA extraction and PCR amplification}

The stored fecal specimens were washed three times with distilled water and genomic DNA was extracted by using an E.Z.N.A. Stool DNA kit (Omega Biotek Inc., Norcross, GA, USA) according to the procedure recommended by the manufacturer. The extracted DNA was stored at $-20{ }^{\circ} \mathrm{C}$ before it was used for PCR amplification. DNA from each specimen was tested at least three times for E. bieneusi DNA using a nested PCR assay that amplified a $392 \mathrm{bp}$ product that encompassed the terminal part of $18 \mathrm{~S}$ rRNA gene, the complete internal transcribed spacer (ITS), and a partial region of $5.8 \mathrm{~S}$ rRNA gene [15]. The KOD-Plus-Neo amplification enzyme (Toyobo Co. Ltd., Osaka, Japan) was used for PCR amplification. Nonacetylated bovine serum albumin (Solarbio Co. Ltd., Beijing, China) (final concentration $400 \mathrm{ng} / \mathrm{L}$ ) was used to neutralize PCR inhibitors during the primary PCR. The secondary PCR products were examined by agarose gel electrophoresis and visualized after GelRed (Biotium Inc., Hayward, CA) staining.

\section{Sequencing and phylogenetic analysis}

Since some fecal samples were collected from the same animals, positive specimens from different farms, animals, species and genders were selected for sequencing. The selected secondary PCR products were purified using Montage PCR filters (Millipore, Bedford, MA) and sequenced using a BigDye Terminator v3.1 cycle sequencing kit (Applied Biosystems) on an ABI 3730 DNA analyzer (Applied Biosystems, Foster City, CA). The nucleotide sequences were confirmed by bidirectional sequencing and by sequencing a new PCR product if necessary. The sequences obtained were aligned with reference sequences downloaded from GenBank using the ClustalX 2.0 (http://www.clustal.org/) program to determine the genotypes. Genotypes from this study were compared with known $E$. bieneusi ITS genotypes by neighbor-joining analysis of the aligned $E$. bieneusi sequences using Mega 5.0 (http://www.megasoftware.net/). Bootstrap analysis was used to assess the robustness of clusters using 1000 replicates. A previously established nomenclature system was used for naming the E. bieneusi ITS genotypes [24].

\section{Statistical analysis}

Infection rates were compared using the chi-square test and a difference was considered statistically significant when the $P$ value was $<0.05$. The analysis was done using QuickCalcs software (GraphPad Software Inc., La Jolla, CA).

\section{Nucleotide sequence accession numbers}

Representative nucleotide sequences from this study have been deposited in GenBank under accession numbers KP262357-KP262398.

\section{Results}

Prevalence of $E$. bieneusi in goats and sheep

Of the 1025 fecal specimens from goats and sheep, overall 353 specimens $(34.4 \%)$ were E. bieneusi-positive by PCR amplification of the ITS locus. The prevalence in goats was $28.8 \%(176 / 611)$ and in sheep was $42.8 \%$ (177/414). The differences in the infection rates between sheep and goats were statistically significant different $\left(x^{2}=21.27, p<0.01\right)$. For goats, E. bieneusi was found on 17 of the studied farms from four provinces and one city (Table 1 and Additional file 1). The prevalence ranged from $7.5 \%$ to $62.5 \%$ for each goat farm, with significant differences in prevalence between different goat farms $\left(X^{2}=73.21, p<0.01\right)$. The prevalence rates were $62.5 \%$ in goats from Chongqing City, $47.8 \%$ in Shaanxi Province, 32.9 \% in Henan Province, $22.4 \%$ in Yunnan Province, and $7.5 \%$ in goats from Anhui Province $\left(x^{2}=35.81, p<0.01\right)$. For sheep, E. bieneusi was detected in six farms from three provinces. The prevalence varied from $9.4 \%$ to $67.4 \%$ in the studied sheep farms, and the differences in infection rates were statistically significant $\left(X^{2}=67.84, p<0.01\right)$ (Table 1 and Additional file 1). The prevalence were $51.9 \%$, $25.0 \%$, and $9.4 \%$ in sheep from Henan Province, Heilongjiang Province, and Liaoning Province, respectively $\left(\chi^{2}=44.96, p<0.01\right)$ (Table 1$)$.

There were no statistically significant differences in the prevalence among the age groups in goats $\left(\chi^{2}=4.50\right.$, $p>0.05)$ and sheep $\left(x^{2}=5.61, p>0.05\right)$ (Table 3). Likewise, there were no statistically significant differences for gender in goats $\left(\chi^{2}=0.01, p>0.05\right)$ and sheep $\left(\chi^{2}=2.05\right.$, $p>0.05$ ) (Table 2). There were some differences in prevalence between grazing and household goats, with a higher prevalence in household goats $\left(x^{2}=5.75,0.01<p<0.05\right)$ (Table 2), but there was no significant difference in prevalence for grazing and household sheep $\left(x^{2}=0.65, p>0.05\right)$ (Table 2).

\section{Distribution of $E$. bieneusi genotypes in goats and sheep}

A total of 42 ITS genotypes were obtained from 230 successfully sequenced specimens from goats and sheep. Of them, nine genotypes have been previously reported, including BEB6 (EU153584), D (AF101200, syn. CEbC; PigEBITS9; VI; WL18; Peru 9; PtEb), EbpC (AF135832, syn. E; WL13; Peru4; WL17), F (AF135833, syn. EbpA), KIN-1(JQ437573), J(AF135837), COS-I(KJ850432, syn. CM7), CM4(KF543866) and CD6(KJ668733), while other 33 genotypes were novel (CHG1-CHG3, CHG5CHG14, CHG16-CHG25 and CHS3-CHS12). A higher sequence polymorphism was identified in goat isolates compared to sheep specimens. Of the 139 goat isolates that were successfully sequenced, 31 genotypes were identified, eight of which were known (BEB6, D, EbpC, F, KIN-1, J, COS-I and CD6), while other 23 were new 
Table 2 Prevalence and genotype distribution of E. bieneusi by age, gender, and feeding habits in goats and sheep

\begin{tabular}{|c|c|c|c|c|c|c|c|c|}
\hline \multicolumn{3}{|c|}{ Age group } & \multirow{3}{*}{$\begin{array}{l}<3 \mathrm{~m} \\
(\mathrm{~b} / \mathrm{c}) \mathrm{d}) \\
6 / 36 \\
(16.7)\end{array}$} & \multirow{3}{*}{$\begin{array}{l}3-6 \mathrm{~m} \\
(\mathrm{~b} / \mathrm{c} d) \\
16 / 35 \\
(45.7)\end{array}$} & \multirow{3}{*}{$\begin{array}{l}6 \mathrm{~m}-1 \mathrm{y} \\
(\mathrm{b} / \mathrm{c} \mathrm{d}) \\
14 / 49 \\
(28.6)\end{array}$} & \multirow{3}{*}{$\begin{array}{l}>1 y \\
(b / c d) \\
59 / 212 \\
(27.8)\end{array}$} & \multirow{3}{*}{$\begin{array}{l}\text { Subtotal } \\
\text { (b/cd) } \\
95 / 332 \\
(28.6)\end{array}$} & \multirow{3}{*}{$\begin{array}{l}\text { Genotypes }\left(n^{a}\right) \\
\text { BEB6(23), D(2), E(6), F(2), J(1), KIN-1(1), CD6(7), COS-I(1), } \\
\text { CHG1(13),CHG2(2), CHG3(10),CHG5(6), CHG6(1), CHG9(1) } \\
\text { CHG11(1), CHG13(1), CHG14(1), CHG16(2), CHG18(1), } \\
\text { CHG19(1), CHG23(1), CHG24(1), CHG25(1); }\end{array}$} \\
\hline \multirow[t]{8}{*}{ Goats } & \multirow[t]{4}{*}{ Gender } & \multirow[t]{2}{*}{ ㅇ } & & & & & & \\
\hline & & & & & & & & \\
\hline & & \multirow[t]{2}{*}{$\hat{0}$} & $12 / 46$ & $7 / 26$ & $3 / 16$ & $59 / 191$ & $81 / 279$ & \multirow{2}{*}{$\begin{array}{l}\mathrm{BEB6}(18), \mathrm{D}(1), \mathrm{E}(2), \mathrm{F}(2), \mathrm{KIN}-1(1), \mathrm{CD} 6(2), \mathrm{COS}-\mathrm{I}(1) \\
\mathrm{CHG1}(6), \mathrm{CHG} 2(4), \mathrm{CHG} 3(7), \mathrm{CHG} 5(1), \mathrm{CHG} \text { (1), CHG8(1), } \\
\text { CHG10(1), CHG12(1), CHG17(1), CHG20(1), CHG21(1), } \\
\text { CHG22(1); }\end{array}$} \\
\hline & & & $(26.1)$ & $(26.9)$ & $(18.8)$ & $(30.9)$ & $(29.0)$ & \\
\hline & \multirow{4}{*}{$\begin{array}{l}\text { Feeding } \\
\text { habits }\end{array}$} & \multirow[t]{2}{*}{ Grazing } & $5 / 24$ & $0 / 0$ & $7 / 34$ & $61 / 242$ & 73/300 & \multirow{2}{*}{$\begin{array}{l}\text { BEB6(21), D(3), E(7), F(2), J(1), COS-I(1), CD6(5), CHG1(5), } \\
\text { CHG2(1), CHG3(12), CHG5(3), CHG16(1), CHG17(1), } \\
\text { CHG19(1), CHG21(1), CHG22(1), CHG25(1); }\end{array}$} \\
\hline & & & (20.8) & (0) & (20.6) & $(25.2)$ & (24.3) & \\
\hline & & \multirow[t]{2}{*}{ Household } & $13 / 58$ & $23 / 61$ & $10 / 31$ & $57 / 161$ & $103 / 311$ & \multirow{2}{*}{$\begin{array}{l}\mathrm{BEB} 6(20), \mathrm{E}(1), \mathrm{F}(1), \mathrm{KIN}-1(2), \mathrm{COS}-\mathrm{I}(1), \mathrm{CD} 6(4), \mathrm{CHG} 1(14) \text {, } \\
\mathrm{CHG} 2(5), \mathrm{CHG} 3(5), \mathrm{CHG} 5(4), \mathrm{CHG} \text { (1), CHG7(1), CHG8(1), } \\
\mathrm{CHG} \text { (1), CHG10(1), CHG11(1), CHG12(1), CHG13(1) } \\
\text {,CHG14(1), CHG16(1), CHG18(1), CHG20(1), CHG23(1), } \\
\mathrm{CHG} 24(1) ;\end{array}$} \\
\hline & & & $(22.4)$ & $(37.7)$ & (32.3) & (35.4) & (33.1) & \\
\hline \multirow[t]{8}{*}{ sheep } & \multirow[t]{4}{*}{ Gender } & \multirow[t]{2}{*}{ 우 } & $0 / 0$ & $26 / 45$ & $2 / 9$ & $74 / 172$ & $106 / 226$ & \multirow{2}{*}{ 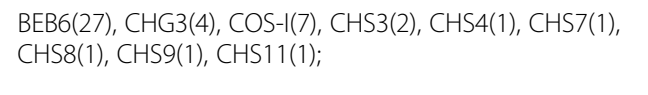 } \\
\hline & & & (0) & $(57.8)$ & $(22.2)$ & $(43.0)$ & (46.9) & \\
\hline & & \multirow[t]{2}{*}{$0^{\lambda}$} & $13 / 25$ & $19 / 41$ & $18 / 43$ & $25 / 79$ & 75/188 & \multirow{2}{*}{$\begin{array}{l}\text { BEB6(33), CM4(1), COS-I(7), CHG3(1), CHS5(1), CHS6(1), } \\
\text { CHS10(1), CHS12(1); }\end{array}$} \\
\hline & & & (52.0) & $(46.3)$ & $(45.9)$ & (31.7) & (39.9) & \\
\hline & \multirow{4}{*}{$\begin{array}{l}\text { Feeding } \\
\text { habits }\end{array}$} & \multirow[t]{2}{*}{ Grazing } & $0 / 0$ & $15 / 21$ & $11 / 22$ & $84 / 205$ & $110 / 248$ & \multirow[t]{2}{*}{$\operatorname{BEB6}(17), \operatorname{COS}-1(5), \mathrm{CM} 4(1), \mathrm{CHG} 3(5), \mathrm{CHS} 4(1) ;$} \\
\hline & & & (0) & $(71.4)$ & $(50.0)$ & $(41.0)$ & $(44.4)$ & \\
\hline & & \multirow[t]{2}{*}{ Household } & $13 / 25$ & $30 / 65$ & $9 / 30$ & $15 / 46$ & $67 / 166$ & \multirow{2}{*}{ 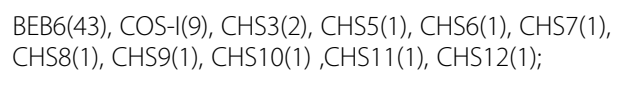 } \\
\hline & & & $(52.0)$ & $(46.2)$ & $(30.0)$ & $(32.6)$ & $(40.4)$ & \\
\hline
\end{tabular}

$n^{\mathrm{a}}$, number of genotypes; + : female sign; $\delta^{2}$ : male

b/c, No.positive/ No.examined; d, \%

genotypes (CHG1-CHG3, CHG5-CHG14, CHG16CHG25). For sheep, 91 specimens were sequenced successfully and 14 genotypes were identified, including three known genotypes (BEB6, CM4 and COS-I) and 11 novel genotypes (CHG3 and CHS3-CHS12). Genotypes BEB6, COS-I and CHG3 were found in both sheep and goat specimens (Tables 1, 2, 3).

The genotype BEB6 was the dominant genotype in goat (41/139) and sheep isolates (60/91) in this study. In goats, in addition to BEB6, the other dominant genotypes, namely $\mathrm{CHG} 1$ and $\mathrm{CHG} 3$, were detected in 19 and 17 specimens, respectively. The genotypes CD6, CHG5, EbpC, CHG2, F, and D were identified in nine, seven, eight, six, four, and three specimens, respectively. Genotypes KIN-1, COS-I and CHG16 were observed in two specimens each, while all other genotypes we identified were found in one specimen each. In sheep, the genotype COS-I was the second dominant genotype, being identified in 14 specimens. Genotypes CHG3 and CHS2 were identified in five and two specimens, respectively, while all other genotypes we obtained were identified in one specimen each. The genotype distribution according to the age, gender and the feeding habits of the animals is shown in Tables 2 and 3.

\section{Phylogenetic analysis}

Phylogenetic analysis revealed that some novel genotypes from this study belonged to the human-pathogenic group [15], recently renamed group $1[39,40]$. We identified genotypes D, KIN-1, EbpC and F reported previously and 10 novel genotypes (CHG6, CHG7, CHG9, CHG19, CHG23, CHG25, CHS5, CHS10, CHS11 and CHS12) belonged to this group. The novel genotypes CHG23, CHG25, CHS10 and CHG19 had one (C to A substitution at nucleotide position 362), two ( $\mathrm{T}$ to $\mathrm{C}$ and $\mathrm{T}$ to A substitutions at positions 243 and 245), two ( $\mathrm{T}$ to $\mathrm{G}$ substitutions at positions 223 and 252) and three (A to G, T to G and G to A substitutions at positions 137, 201 and 374) single nucleotide polymorphisms (SNPs) compared with the established genotype, EbpC (AF135832). Therefore, genotypes CHG23, CHG25, NESH1, CHS10 and CHG19 were clustered into subgroup $1 \mathrm{~d}$ with the $\mathrm{EbpC}$ genotype. The following new genotypes CHS5, CHS11 and CHS12 were grouped with subgroup 1e with known genotypes COG-1 (KJ850434) and F (AF135833). Compared with the known genotype F (AF135833), one or several SNPs were present (data not shown). The new genotype CHG9 differed from the genotype C (AF101199) at nucleotide positions 123 ( $\mathrm{G}$ to A substitution), 230 (G to A substitution), 255(G to $\mathrm{T}$ substitution) and 284 (G to A substitution), 
Table 3 Prevalence and ITS genotype distribution of E. bieneusi isolates by age group in goats and sheep

\begin{tabular}{|c|c|c|c|}
\hline Species & Age group & $\begin{array}{l}\text { (No. positive/ } \\
\text { No.examined) (\%) }\end{array}$ & Genotypes $\left(n^{a}\right)$ \\
\hline \multirow[t]{5}{*}{ Goats } & $<3 \mathrm{~m}$ & 18/82(22.0) & CHG1(1), CHG2(1), CD6(4), CHG5(1); \\
\hline & $3-6 m$ & 23/61(37.7) & $\mathrm{BEB} 6(4), \mathrm{F}(1), \mathrm{CD} 6(2), \mathrm{CHG} 1(5), \mathrm{CHG} 2(2), \mathrm{CHG} 3(3), \mathrm{CHG} 5(2), \mathrm{CHG} 14(1)$; \\
\hline & $6 \mathrm{~m}-1 \mathrm{y}$ & $17 / 65(26.2)$ & BEB6(10), E(2), CHG13(1), CHG18(1); \\
\hline & $>1 y$ & 118/403(29.3) & $\begin{array}{l}\mathrm{BEB} 6(27), \mathrm{D}(3), \mathrm{E}(6), \mathrm{F}(3), \mathrm{J}(1), \mathrm{KIN}-1(2), \mathrm{CD} 6(3), \mathrm{COS}-\mathrm{I}(2), \mathrm{CHG} 1(13), \mathrm{CHG} \text { (3), CHG3(14), CHG5(4), CHG6(1), } \\
\mathrm{CHG7}(1), \mathrm{CHG}(1), \mathrm{CHG} 9(1), \mathrm{CHG10}(1), \mathrm{CHG} 11(1), \mathrm{CHG} 12(1), \mathrm{CHG} 16(2), \mathrm{CHG} 17(1), \mathrm{CHG} 19(1), \mathrm{CHG} 20(1), \\
\mathrm{CHG} 21(1), \mathrm{CHG} 22(1), \mathrm{CHG} 23(1), \mathrm{CHG} 24(1), \mathrm{CHG} 25(1) ;\end{array}$ \\
\hline & Subtotal & 176/611(28.8) & $\begin{array}{l}\text { BEB6(41), D(3), E(8), F(4), KIN-1(2), J(1), CD6(9), COS-I(2), CHG1(19), CHG2(6), CHG3(17), CHG5(7), CHG6(1), } \\
\text { CHG7(1), CHG8(1),CHG9(1), CHG10(1), CHG11(1), CHG12(1), CHG13(1), CHG14(1), CHG16(2), CHG17(1), } \\
\text { CHG18(1), CHG19(1), CHG2O(1), CHG21(1), CHG22(1), CHG23(1), CHG24(1), CHG25(1); }\end{array}$ \\
\hline \multirow[t]{5}{*}{ Sheep } & $<3 \mathrm{~m}$ & $13 / 25(52.0)$ & BFB6(9), CHS6(1), COS-I(2); \\
\hline & $3-6 \mathrm{~m}$ & 45/86(52.3) & BFB6(26), COS-I(6), CHG3(2), CHS3(1), CHS4(1), CHS5(1), CHS7(1), CHS8(1), CHS9(1), CHS11(1); \\
\hline & $6 \mathrm{~m}-1 \mathrm{y}$ & 20/52(38.5) & $\mathrm{BEB6}(7), \mathrm{CHS10}(1), \mathrm{CHS12}(1)$ \\
\hline & $>1 y$ & 99/251(39.4) & BEB6(18), CM4(1), COS-I(6) ,CHG3(3), CHS3(1); \\
\hline & Subtotal & $177 / 414(42.8)$ & $\begin{array}{l}\mathrm{BEB} 6(60), \mathrm{CHG} 3(5), \mathrm{COS}-\mathrm{I}(14), \mathrm{CM} 4(1), \mathrm{CHS} 3(2), \mathrm{CHS} 4(1), \mathrm{CHS5} \text { (1), CHS6(1), CHS7(1), CHS8(1), } \\
\mathrm{CHS9} 9 \text { (1), CHS10(1), CHS11(1),CHS12(1); }\end{array}$ \\
\hline Total & & $353 / 1025(34.4)$ & $\begin{array}{l}\text { F(4), KIN-1(2), J(1), CHG1(19), CHG2(6), CHG3(22), CD6(9), CHG5(7), CHG6(1), CHG7(1), CHG8(1), CHG9(1), } \\
\text { CHG10(1),CHG11(1),CHG12(1), CHG13(1), CHG14(1), COS-I(16), CHG16(2), CHG17(1), CHG18(1), CHG19(1), } \\
\text { CHG20(1), CHG21(1), CHG22(1),CHG24(1),CHG25(1),CM4(1), CHS3(2), CHS4(1), CHS5(1), CHS6(1), CHS7(1), } \\
\text { CHS8(1), CHS9(1), CHS10(1), CHS11(1), CHS12(1),BEB6(101), D(3), E(8), CHG23(1) }\end{array}$ \\
\hline
\end{tabular}

$n^{\text {a }}$, number of genotypes

respectively, thus they formed subgroup $1 \mathrm{f}$. However, the new genotypes CHG6 and CHG7 formed a cluster distinct from any known subgroup 1 . We found that 22 new genotypes (CHG1-CHG3, CHG5, CHG8, CHG10-CHG14, CHG16-CHG18, CHG20, CHG22, CHG24, CHS3, CHS4, and CHS6-CHS9) and 7 known genotypes (CD6, COS-I, BEB6, J, COS-III, NESH4 and CHN2) belonged to the host-specific group 2, but the known genotypes J (AF135837) and CHN2 (HM992510) formed one branch, and the 22 new genotypes with the reported genotypes CD6(KJ668733), COS-I(KJ850432),COS-III(KP063053), NESH4(KP732478) and BEB6(EU153584) formed another clade. Compared with genotype BEB6 (EU153584), new genotypes CHG2, CHG13, CHG16, CHG17, CHS3, CHS4, CHS7, CHS8 and CHS9 contained one SNP, genotypes CHG3, CHG5, CHG12, CHG22 and CHS6 contained two SNPs, genotypes CHG1, CHG14, CHG18 and CHG20 contained three SNPs, and genotypes CHG11, CHG10 and CHG24 contained 4, 11 and 13 SNPs, respectively, the genotype CHG8 contained over 15 SNPs (Additional file 2). However, the new genotype CHG21 was clustered with the CM4 (KF543866) genotype [20, this study] in the group 9 [41] ( Additional file 3: Figure S1).

\section{Discussion}

In the present study, E. bieneusi was found to be a common parasite in sheep and goats. The prevalence of this organism in healthy sheep and goats was $42.8 \%$ and $28.8 \%$, respectively. A similar prevalence $(21.8 \%)$ was reported for E. bieneusi in goats in Heilongjiang
Province [37], while lower prevalence rates $(22.5 \%$ and $13.9 \%)$ were detected in Heilongjiang Province [37] and northeast China [35] in sheep. A higher E. bieneusi prevalence $(69.3 \%)$ was found in sheep in Inner Mongolia [34]. The differences in the prevalence between this study and others $[6,9,34,35,37]$ are likely due to differences in the age groups studied, as well as the number of study animals, detection methods, and the geographic and ecological environments. Here we described the infection rates of $E$. bieneusi in different age groups in sheep and goats and showed that there were no significant differences among different age groups, which was consistent with the conclusion obtained by Jiang and others [35] but inconsistent with the results gained by Stensvold [10]. In this study, we have described the prevalence of $E$. bieneusi by the gender and different feeding habits of the host animals for the first time in detail, although there were no significant differences between them. Nevertheless, these results provide an useful reference data for researchers in this field.

All nine known genotypes identified in the present study have been previously reported in China: the genotype BEB6 has been reported in one child (reported as SH5, [30]), sheep [9], NHPs [20, 42], deer [36], cats [12] and golden takins [43], even in urban wastewater [38]. This genotype was first detected in cattle in the United States [13] and is considered to be a cattle-adopted genotype. However, the genotype BEB6 was detected in $43.9 \%(101 / 230)$ sequenced specimens and found in almost every farm (Additional file 1), which showed that 
this genotype was observed as a dominant genotype with a wide geographic distribution, extensive host range and zoonotic potential both in the present study and in other reports [9, 10, 23, 34-37]. The genotype D has been found in AIDS patients [30], children [31], NHPs [33, 42], pigs [8], dogs [12, 44], cats [12, 44], foxes [45], raccoon dogs [45], cattle [46], golden takins [43], urban wastewater [38] and drinking source watershed [47]. In this report, this genotype was only found in goats, in contrast to the results reported in Heilongiiang Province [37]. Though the genotype D only infected goats in this study, the transmission source and transmission dynamics of this genotype is still unclear and further research is required to understand the role of this genotype in microsporidiosis. The genotype EbpC has been found in HIV-positive and -negative patients [30], children [31], NHPs [20,33], dogs [12, 44], pigs $[8,9]$, lake water [32], red pandas [48] and urban wastewater [38]. Here, the genotype $\mathrm{EbpC}$ was detected only in goat specimens, which was similar to the results of Zhao et al [37], but was in contrast with Li et al [35]. The genotype $\mathrm{F}$ has been found in children [31], pigs [8], cattle [46] and wastewater [38]. Genotypes J has been reported in humans [11], cattle [49] and yaks [50], KIN-1 in humans (JQ437573, unpublished), CD6(KJ668733), COSI(KJ850432) (syn. CM7) in sheep [35, 37], deer [51] and NHPs [20], CM4 in NHPs [20] and CD6 in dogs [20].

Among the nine established genotypes found in this study, six (BEB6, D, EbpC, F, KIN-1, J) genotypes have been reported in humans $[11,30,31]$. Thus, some of the genotypes in sheep and goats have zoonotic potential, and sheep and goats may be reservoirs of human microsporidiosis.

Phylogenetic analysis showed that 10 new E. bieneusi genotypes (CHG6, CHG7, CHG9, CHG19, CHG23, CHG25, CHS5, CHS10, CHS11, and CHS12) belonged to the so-called group 1 with zoonotic potential [39]. This finding merits close attention because of the ability of these genotypes to cause E. bieneusi-related microsporidiosis in humans. The other 22 new genotypes (CHG1CHG3, CHG5, CHG8, CHG10-CHG14, CHG16-CHG18, CHG20, CHG22, CHG24, CHS3, CHS4, and CHS6CHS9) were classified into the cattle-specific group 2 [39]. Some genotypes from this group initially considered to be cattle-specific (such as BEB6 and J), were later found in other animals including humans $[11,12,20,30,50$, this research], therefore some genotypes in the group 2 may have limited zoonotic potential and should be examined in more detail. The remaining new genotype CHG21 with only one SNP compared with the genotype CM4 formed a cluster, in the previously described group 9 [41].

\section{Conclusions}

This study to infer the prevalence and genotype distribution of E. bieneusi in sheep and goats in China. The data showed there were a widespread distribution, high prevalence, and considerable genetic diversity in E. bieneusi genotypes from farmed goats and sheep, which may cause microsporidiosis among sheep, goats, other animals and humans resulting in economic losses in animal husbandry. Moreover, many genotypes from this study have been reported in humans and urban wastewater in China, which may result in zoonotic transmission. Therefore, contact between susceptible human populations and goats or sheep should be avoided to reduce zoonotic or anthroponotic parasite transmission. Further studies are needed to fully elucidate the importance of goats and sheep in the epidemiology of human microsporidiosis.

\section{Additional files}

Additional file 1: Prevalence and genotype distribution of $E$. bieneusi in goats and sheep on each farm. (DOC $117 \mathrm{~kb}$ )

Additional file 2: Nucleotide substitutions among some new genotypes of $E$. bieneusi from this study versus the reported genotype BEB6 (EU 153584). (DOC $132 \mathrm{~kb}$ )

Additional file 3: Figure S1. FIG 1 Phylogenetic relationships of the $E$. bieneusi genotypes identified in this study and other reported genotypes, as inferred by neighbor-joining analysis of ITS gene sequences based on the distances calculated using the Kimura 2-parameter model. Bootstrap values $>50 \%$ from 1000 replicates are shown on the nodes. The tree was rooted with GenBank sequence AF059610 from a dog. Known genotypes observed in goats and sheep are marked with open squares and triangles, and the new genotypes in goats and sheep from this study are indicated by filled squares and triangles. (TIF $2078 \mathrm{~kb}$ )

\section{Competing interests}

The authors declare that they have no competing interests.

\section{Authors' contributions}

CSN conceived and designed the experiments; KS, MJL, and XXW performed the experiments; KS, JQL and MRK analyzed the data; KS, RJW, LXZ, FCJ and CSN wrote the manuscript. All the authors have read and approved the final version of the manuscript.

\section{Acknowledgments}

This work was supported by the Earmarked Fund for China Modern Agroindustry Technology Research System (nycytx-39) and the Collaborative Innovation Center of Modern Animal Husbandry, Henan Province, China. We thanks for Professor Una Ryan from School of Veterinary and Life Sciences, Murdoch University, Murdoch, Western Australia, to copy-edit our MS.

Received: 3 August 2015 Accepted: 10 January 2016

Published online: 19 January 2016

\section{References}

1. Keeling PJ, Fast NM. Microsporidia: biology and evolution of highly reduced intracellular parasites. Annu Rev Microbiol. 2002;56:93-116.

2. Keeling P. Five questions about microsporidia. PLoS Pathog. 2009;5: e1000489.

3. Santín M, Fayer R. Microsporidiosis: Enterocytozoon bieneusi in domesticated and wild animals. Res Vet Sci. 2011;90:363-71.

4. Desportes I, Le Charpentier Y, Galian A, Bernard F, Cochand-Priollet B, Lavergne A, et al. Occurrence of a new microsporidan: Enterocytozoon bieneusi n. g., n. sp., in the enterocytes of a human patient with AIDS. J Protozool. 1985:32:250-4.

5. Didier ES. Microsporidiosis: an emerging and opportunistic infection in humans and animals. Acta Trop. 2005;94:61-76.

6. Lores B, del Aguila C, Arias C. Enterocytozoon bieneusi (Microsporidia) in faecal specimen from domestic animals from Galicia, Spain. Mem Inst Oswaldo Cruz. 2002;97:941-5. 
7. Del Aguila C, Izquierdo F, Navajas R, Pieniazek NJ, Miró G, Alonso Al, et al. Enterocytozoon bieneusi in animals: rabbits and dogs as new hosts. J Eukaryot Microbiol. 1999;46:8S-9S.

8. Li W, Diao R, Yang J, Xiao L, Lu Y, Li Y, et al. High diversity of human-pathogenic Enterocytozoon bieneusi genotypes in swine in northeast China. Parasitol Res. 2014;113:1147-53.

9. $\quad$ Li W, Li Y, Li W, Yang J, Song M, Diao R, et al. Genotypes of Enterocytozoon bieneusi in livestock in China: high prevalence and zoonotic potential. PLoS One. 2014;9:e97623.

10. Stensvold CR, Beser J, Ljungström B, Troell K, Lebbad M. Low host-specific Enterocytozoon bieneusi genotype BEB6 is common in Swedish lambs. Vet Parasitol. 2014;205:371-4.

11. Zhang $X$, Wang $Z$, Su Y, Liang $X$, Sun $X$, Peng $S$, et al. Identification and genotyping of Enterocytozoon bieneusi in China. J Clin Microbiol. 2011:49:2006-8.

12. Karim MR, Dong H, Yu F, Jian F, Zhang L, Wang R, et al. Genetic diversity in Enterocytozoon bieneusi isolates from dogs and cats in China: host specificity and public health implications. J Clin Microbiol. 2014;52:3297-302.

13. Fayer R, Santin M, Trout JM. Enterocytozoon bieneusi in mature dairy cattle on farms in the eastern United States. Parasitol Res. 2007;102:15-20.

14. Wagnerová $P$, Sak $B$, Květoňová $D$, Buňatová $Z$, Civišová $H$, Maršálek $M$, et al Enterocytozoon bieneusi and Encephalitozoon cuniculi in horses kept under different management systems in the Czech Republic. Vet Parasitol. 2012;190:573-7.

15. Sulaiman IM, Fayer R, Lal AA, Trout JM, Schaefer 3rd FW, Xiao L. Molecular characterization of microsporidia indicates that wild mammals harbor hostadapted Enterocytozoon spp. as well as human-pathogenic Enterocytozoon bieneusi. Appl Environ Microbiol. 2003:69:4495-501.

16. Dengjel B, Zahler M, Hermanns W, Heinritzi K, Spillmann T, Thomschke A, et al. Zoonotic potential of Enterocytozoon bieneusi. J Clin Microbiol. 2001:39:4495-9.

17. Drosten C, Laabs J, Kuhn EM, Schottelius J. Interspecies transmission of Enterozytozoon bieneusi supported by observations in laboratory animals and phylogeny. Med Microbiol Immunol. 2005;194:207-9.

18. Cama VA, Pearson J, Cabrera L, Pacheco L, Gilman R, Meyer S, et al. Transmission of Enterocytozoon bieneusi between a child and guinea pigs. J Clin Microbiol. 2007:45:2708-10.

19. Santín M, Fayer R. Enterocytozoon bieneusi, Giardia, and Cryptosporidium infecting white-tailed deer. J Eukaryot Microbiol. 2014;194:207-9.

20. Karim MR, Wang R, Dong H, Zhang L, Li J, Zhang S, et al. Genetic polymorphism and zoonotic potential of Enterocytozoon bieneusi from nonhuman primates in China. Appl Environ Microbiol. 2014;80:1893-8.

21. Karim MR, Yu F, Li J, Li J, Zhang L, Wang R, et al. First molecular characterization of enteric protozoa and the human pathogenic microsporidian, Enterocytozoon bieneusi, in captive snakes in China. Parasitol Res. 2014;113:3041-8.

22. Haro M, Izquierdo F, Henriques-Gil N, Andrés I, Alonso F, Fenoy S, et al. First detection and genotyping of human-associated microsporidia in pigeons from urban parks. Appl Environ Microbiol. 2005;71:3153-7.

23. Feng Y, Li N, Dearen T, Lobo ML, Matos O, Cama V, et al. Development of a multilocus sequence typing tool for high-resolution genotyping of Enterocytozoon bieneusi. Appl Environ Microbiol. 2011;77:4822-8.

24. Santín M, Fayer R. Enterocytozoon bieneusi genotype nomenclature based on the internal transcribed spacer sequence: a consensus. J Eukaryot Microbiol. 2009:56:34-8.

25. Lobo ML, Xiao L, Cama V, Stevens T, Antunes F, Matos O. Genotypes of Enterocytozoon bieneusi in mammals in Portugal. J Eukaryot Microbiol. 2006;53:561-4

26. Santín M, Trout JM, Fayer R. Enterocytozoon bieneusi genotypes in dairy cattle in the eastern United States. Parasitol Res. 2005;97:535-8.

27. Santín M, Trout JM, Vecino JA, Dubey JP, Fayer R. Cryptosporidium, Giardia and Enterocytozoon bieneusi in cats from Bogota (Colombia) and genotyping of isolates. Vet Parasitol. 2006;141:334-9.

28. Sulaiman IM, Bern C, Gilman R, Cama V, Kawai V, Vargas D, et al. A molecular biologic study of Enterocytozoon bieneusi in HIV infected patients in Lima, Peru. J Eukaryot Microbiol. 2003;50:591-6.

29. Abe N, Kimata I, Iseki M. Molecular evidence of Enterocytozoon bieneusi in Japan. J Vet Med Sci. 2009:71:217-9.

30. Wang L, Zhang H, Zhao X, Zhang L, Zhang G, Guo M, et al. Zoonotic Cryptosporidium species and Enterocytozoon bieneusi genotypes in HIVpositive patients on antiretroviral therapy. J Clin Microbiol. 2013;51:557-63.

31. Wang L, Xiao L, Duan L, Ye J, Guo Y, Guo M, et al. Concurrent infections of Giardia duodenalis, Enterocytozoon bieneusi, and Clostridium difficile in children during a cryptosporidiosis outbreak in a pediatric hospital in China. PLoS Negl Trop Dis. 2013;7:e2437.

32. Ye J, Xiao L, Ma J, Guo M, Liu L, Feng Y. Anthroponotic enteric parasites in monkeys in public park, China. Emerg Infect Dis. 2012;18:1640-3.

33. Ye J, Xiao L, Li J, Huang W, Amer SE, Guo Y, et al. Occurrence of human-pathogenic Enterocytozoon bieneusi, Giardia duodenalis and Cryptosporidium genotypes in laboratory macaques in Guangxi, China. Parasitol Int. 2014;63:132-7.

34. Ye J, Xiao L, Wang Y, Guo Y, Roellig DM, Feng Y. Dominance of Giardia duodenalis assemblage A and Enterocytozoon bieneusi genotype BEB6 in sheep in Inner Mongolia, China. Vet Parasitol. 2015;210:235-9.

35. Jiang Y, Tao W, Wan Q, Li Q, Yang Y, Lin Y, et al. Zoonotic and potentially host-adapted Enterocytozoon bieneusi genotypes in sheep and cattle in Northeast China and an increasing concern about the zoonotic importance of previously considered ruminant-adapted genotypes. Appl Environ Microbiol. 2015:81:3326-35.

36. Zhao W, Zhang W, Wang R, Liu W, Liu A, Yang D, et al. Enterocytozoon bieneusi in sika deer (cervus nippon) and red deer (cervus elaphus): deer specificity and zoonotic potential of ITS genotypes. Parasitol Res. 2014; 113:4243-50.

37. Zhao W, Zhang W, Yang D, Zhang L, Wang R, Liu A. Prevalence of Enterocytozoon bieneusi and genetic diversity of ITS genotypes in sheep and goats in China. Infect Genet Evol. 2015;32:265-70.

38. Li N, Xiao L, Wang L, Zhao S, Zhao X, Duan L, et al. Molecular surveillance of Cryptosporidium spp., Giardia duodenalis, and Enterocytozoon bieneusi by genotyping and subtyping parasites in wastewater. PLoS Negl Trop Dis. 2012;6:e1809.

39. Thellier M, Breton J. Enterocytozoon bieneusi in human and animals, focus on laboratory identification and molecular epidemiology. Parasite. 2008;15:349-58

40. Henriques-Gil N, Haro M, Izquierdo F, Fenoy S, del Aguila C. Phylogenetic approach to the variability of the microsporidian Enterocytozoon bieneusi and its implications for inter- and intrahost transmission. Appl Environ Microbiol. 2010;76:3333-42.

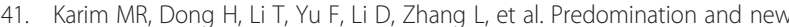
genotypes of Enterocytozoon bieneusi in captive nonhuman primates in zoos in China: high genetic diversity and zoonotic significance. PLoS One. 2015;10:e0117991.

42. Du SZ, Zhao GH, Shao JF, Fang YQ, Tian GR, Zhang LX, et al. Cryptosporidium spp. Giardia intestinalis, and Enterocytozoon bieneusi in Captive NonHuman Primates in Qinling Mountains. Korean J Parasitol. 2015:53:395-402

43. Zhao GH, Du SZ, Wang HB, Hu XF, Deng MJ, Yu SK, et al. First report of zoonotic Cryptosporidium spp., Giardia intestinalis and Enterocytozoon bieneusi in golden takins (Budorcas taxicolor bedfordi). Infect Genet Evol. 2015:34:394-401.

44. Li W, Li Y, Song M, Lu Y, Yang J, Tao W, et al. Prevalence and genetic characteristics of Cryptosporidium, Enterocytozoon bieneusi and Giardia duodenalis in cats and dogs in Heilongjiang province, China. Vet Parasitol. 2015:208:125-34.

45. Yang Y, Lin Y, Li Q, Zhang S, Tao W, Wan Q, et al. Widespread presence of human-pathogenic Enterocytozoon bieneusi genotype D in farmed foxes (Vulpes vulpes) and raccoon dogs (Nyctereutes procyonoides) in China: first identification and zoonotic concern. Parasitol Res. 2015:114:4341-8

46. Zhao W, Zhang W, Yang F, Zhang L, Wang R, Cao J, et al. Enterocytozoon bieneusi in dairy cattle in the Northeast of China: genetic diversity of ITS gene and evaluation of zoonotic transmission potential. J Eukaryot Microbiol. 2015:62:553-60.

47. Guo Y, Alderisio KA, Yang W, Cama V, Feng Y, Xiao L. Host specificity and source of Enterocytozoon bieneusi genotypes in a drinking source watershed. Appl Environ Microbiol. 2014;80:218-25.

48. Tian GR, Zhao GH, Du SZ, Hu XF, Wang HB, Zhang LX, et al. First report of Enterocytozoon bieneusi from giant pandas (Ailuropoda melanoleuca) and red pandas (Ailurus fulgens) in China. Infect Genet Evol. 2015:34:32-5.

49. Ma J, Li P, Zhao X, Xu H, Wu W, Wang Y, et al. Occurrence and molecular characterization of Cryptosporidium spp. and Enterocytozoon bieneusi in dairy cattle, beef cattle and water buffaloes in China. Vet Parasitol. 2015;207:220-7 
50. Ma J, Cai J, Ma J, Feng Y, Xiao L. Enterocytozoon bieneusi genotypes in yaks (Bos grunniens) and their public health potential. J Eukaryot Microbiol. 2015;62:21-5.

51. Zhang Z, Huang J, Karim MR, Zhao J, Dong H, Ai W, et al. Zoonotic Enterocytozoon bieneusi genotypes in Pere David's deer (Elaphurus davidianus) in Henan, China. Exp Parasitol. 2015;155:46-8.

Submit your next manuscript to BioMed Central and we will help you at every step:

- We accept pre-submission inquiries

- Our selector tool helps you to find the most relevant journal

- We provide round the clock customer support

- Convenient online submission

- Thorough peer review

- Inclusion in PubMed and all major indexing services

- Maximum visibility for your research

Submit your manuscript at www.biomedcentral.com/submit 